\title{
Investigation of the Effect of Building Dielectric Parameters on Indoor Radio Channel Characteristics
}

\author{
Y. E. Mohammed \\ University of Mosul/ College of Engineering, Elect. Engg., Dept. \\ a.yessar@yahoo.com
}

\begin{abstract}
The unlicensed industrial, scientific, and medical (ISM) band at $2.4 \mathrm{GHz}$ has gained increased attention recently due to high data rate communication systems developed to operate in this band. In this paper the investigation of the indoor electromagnetic propagation and indoor radio channel characteristics have been performed at this band. Electromagnetic waves propagate by means of reflection, refraction, and diffraction. Reflection mechanisms are dependent on the nature of the surface, dielectric properties, polarization, incidence angle, and material thickness. The effect of the incidence angle and material thickness on the reflection coefficients for both horizontal and vertical polarization has been studied. Twodimensional ray-tracing simulation has been performed to show the influence of building electromagnetic properties on indoor radio channel parameters, signal level, RMS delay spread, and coherence bandwidth. Results show the influence of the permittivity is more important than the influence of the order of reflection considered for the ray- tracing model. It is shown that, compared with power level, RMS delay spread is more sensitive to the building dielectric parameters. Maximum RMS delay spread is dependent on the dielectric parameters of the surrounded walls.
\end{abstract}

Keywords: Radio propagation, Dielectric parameters, Indoor Channel Parameters

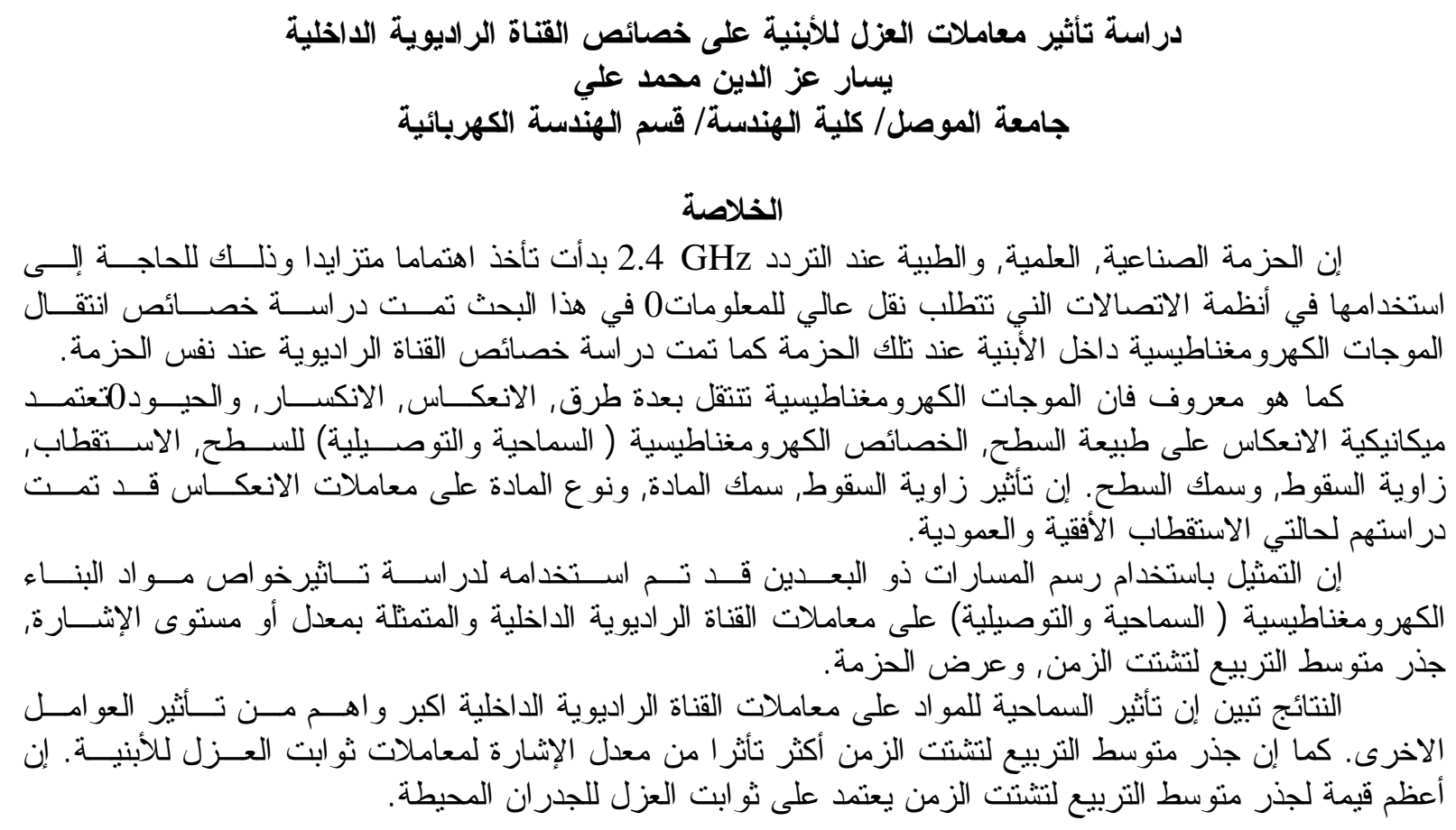




\section{Introduction:}

Analysis of indoor radio channel characteristics is important for the design and development of personal communication systems. Wireless local area networks (WLAN), in particular, have been allocated unlicensed Industrial, Scientific, and Medical (ISM) frequency bands at $2.4 \mathrm{GHz}$. To develop such systems, examination of the mechanisms of electromagnetic wave propagation and the effect of the electromagnetic properties of building materials at this band is required. Radio propagation in the indoor radio channel is affected by the layout of the building especially the use of different building materials. Owing to reflection, refraction and diffraction of radio waves by objects such as walls, windows, doors and furniture inside the building, the direct path from transmitter to receiver is often blocked and there are many additional signal paths present due to reflections off of the walls, floors, and ceilings of the building. Effects of these propagation mechanisms on the radio wave are determined by the electromagnetic properties of those surrounding structures. Therefore to obtain accurate channel characteristics, a good knowledge of their electromagnetic properties is required.

The performance of digital communication systems is strongly dependent on the wideband parameters of the channel, signal level, time dispersion, and coherence bandwidth. For indoor channel characterization, the path loss is a parameter that can predict the power level of the system and the space coverage of the base station, while RMS delay spread is a good measure of channel multipath spread and can give an indication of the potential for inter- symbol interference [1]. The delay spread is therefore an essential parameter in the design of wireless communication systems.

The objective of the study reported here is to characterize the indoor electromagnetic propagation environment at $2.4 \mathrm{GHz}$ ISM band. A model based on multiple successive internal reflections inside the slab has been successfully used to simulate the reflection coefficients as functions of the incident angle and to describe the reflection mechanisms for smooth surfaces with a thickness is not too large compared to the wavelength. The effect of electromagnetic parameters on indoor radio channel characteristics under line-of-sight (LOS) condition has been performed by using two-dimensional ray tracing. It is worth mentioning that the dielectric material properties (permittivity and conductivity) for the materials used in this paper were taken from [2], which are measured at the same band.

\section{Computation of Reflection Coefficients:}

The reflection coefficient from the smooth surfaces can be divided into two types, reflection factor for horizontal polarization $\left(\Gamma_{h}\right)$, and for vertical polarization $\left(\Gamma_{v}\right)$, and they are given by [3]:

$$
\begin{gathered}
\Gamma_{h}=\frac{\sin (\theta)-\sqrt{\varepsilon-\cos ^{2}(\theta)}}{\sin (\theta)+\sqrt{\varepsilon-\cos ^{2}(\theta)}} \\
\Gamma_{v}=\frac{\varepsilon \sin (\theta)-\sqrt{\varepsilon-\cos ^{2}(\theta)}}{\varepsilon \sin (\theta)+\sqrt{\varepsilon-\cos ^{2}(\theta)}}
\end{gathered}
$$

where: $\theta$ is the angle of incidence and $\varepsilon$ is the complex permittivity given by $\varepsilon=\varepsilon_{r}-j 60 \sigma \lambda$ [4], where $\varepsilon_{r}$ is the relative dielectric constant of the reflecting surface, $\sigma$ is the conductivity of the reflecting surface, and $\lambda$ is the wavelength of the incident ray.

When the thickness of the material is not too large compared to the wavelength, the 


\section{Mohammed: Investigation of the Effect of Building Dielectric Parameters on Indoor}

equations (1), and (2) may lead to significant errors. In this case each incident ray to such materials rise to multiple reflected and transmitted rays, and the strength of these rays are functions of the material and its thickness as well as the signal frequency and the angle of incident ray, thus the reflection characteristics of the material can be explained using multi ray model as shown in Fig.1, and the overall reflection coefficients are given by [4]:

$\Gamma_{t}=\Gamma-\left(1-\Gamma^{2}\right) \frac{\Gamma e^{-j 2 k s} e^{-2 \alpha s} e^{j k_{0} d \cos (\theta)}}{1-\Gamma^{2} e^{-j 2 k s} e^{-2 \alpha s} e^{j k_{0} d \cos (\theta)}}$

Where:

$k$ is the propagation constant inside the material

$k=\frac{2 \pi}{\lambda} \sqrt{\varepsilon_{r}}$

$\alpha$ is the attenuation constant inside the material

$\alpha=\frac{\omega \tan (\delta)}{2} \sqrt{\mu_{0} \varepsilon_{r} \varepsilon_{0}} \quad$ (for lossy dielectric)

$k_{0}$ is the free space propagation constants

$k_{0}=\frac{2 \pi}{\lambda}$

$s$ is the path length inside the material between the two surfaces

$s=\frac{l}{\sqrt{1-\frac{\cos ^{2}(\theta)}{\varepsilon_{r}}}}$

$d$ is the path length difference on the material of two consecutive departing reflections

$d=\frac{2 l}{\sqrt{\frac{\varepsilon_{r}}{\cos ^{2}(\theta)}-1}}$

$\Gamma$ is either the horizontal or vertical reflection coefficient as in (1), and (2).

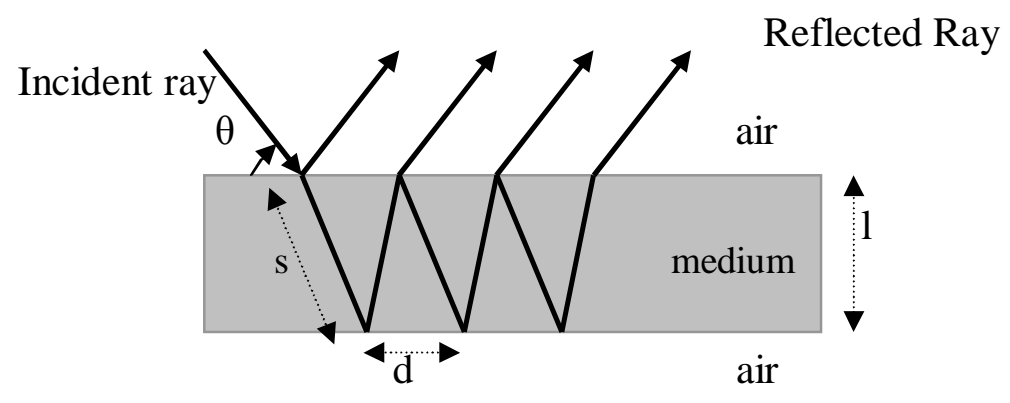

Fig. (1). Reflections from a dielectric material

\section{Reflection Coefficients Characterization:}

Four various types of building materials were used to study the characterization of reflection coefficients as a function of the incidence angle, these materials are, $12 \mathrm{~mm}$ glass, $8 \mathrm{~mm}$ chipwood, $38 \mathrm{~mm}$ thickwood, and $40 \mathrm{~mm}$ door. It is clear that the thickness of the materials is less than the wavelength, thus the multi ray model must be used to simulate the reflection coefficients. The electromagnetic characteristics of the above materials were taken from [2], as shown in table (1). 
Table (1): Material characterization at $2.4 \mathrm{GHz}$

\begin{tabular}{|c|c|c|c|c|}
\hline \multirow{2}{*}{ Material } & \multicolumn{2}{|c|}{$\mathrm{VP}$} & \multicolumn{2}{c|}{$\mathrm{HP}$} \\
\cline { 2 - 5 } & $\varepsilon_{r}$ & $\sigma(\mathrm{S} / \mathrm{m})$ & $\varepsilon_{r}$ & $\sigma(\mathrm{S} / \mathrm{m})$ \\
\hline Glass & 5.73 & 0.088 & 5.70 & 0.073 \\
\hline Chipwood & 2.45 & 0.080 & 2.65 & 0.064 \\
\hline Thickwood & 4.22 & 0.054 & 4.75 & 0.050 \\
\hline Door & 5.91 & 0.030 & 5.63 & 0.045 \\
\hline Concrete wall & 3.52 & 0.028 & 4.12 & 0.014 \\
\hline
\end{tabular}

Fig. (2). Shows the magnitude of the reflection coefficients as a function of incidence angle for both horizontal and vertical polarization for those four materials.

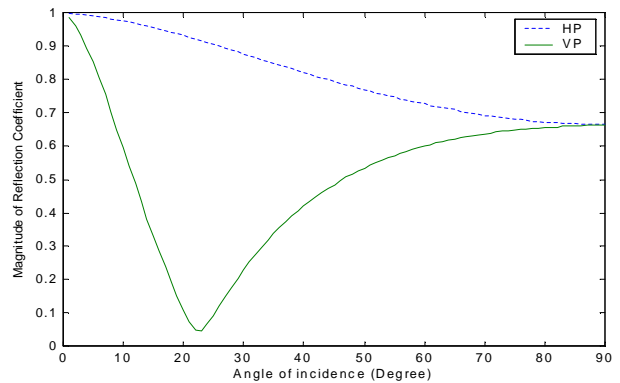

(a)

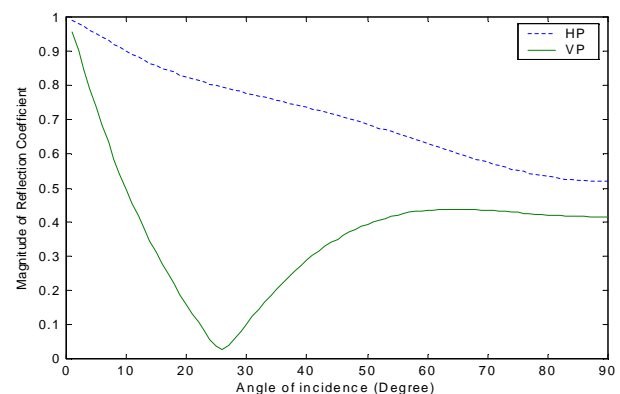

(c)

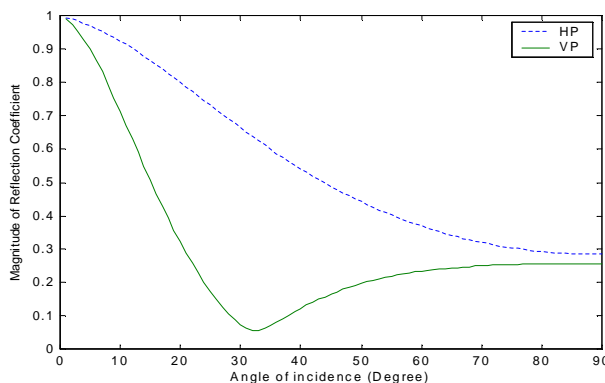

(b)

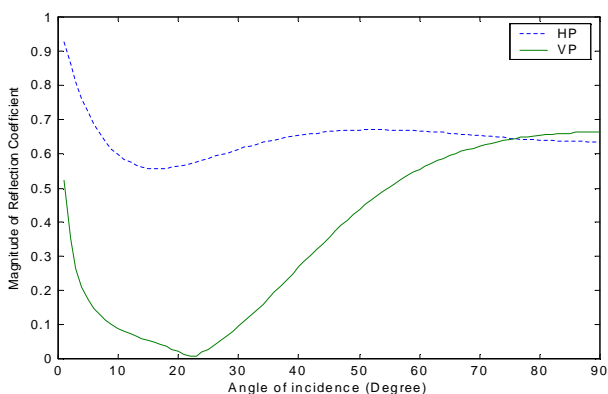

(d)

Fig. (2). Reflection coefficients as a function of incident angle, (a) $12 \mathrm{~mm}$ glass, (b) $8 \mathrm{~mm}$ chipwood, (c) $38 \mathrm{~mm}$ thickwood, (d) 40mm door.

To estimate the degree of reflection coefficient variation, reflection coefficient is simulated as a function of materials thickness for a given values of incidence angle.

Fig. (3). Shows reflection coefficient variations for glass at 10, and 50 degree incident angle respectively for both horizontal and vertical polarization.

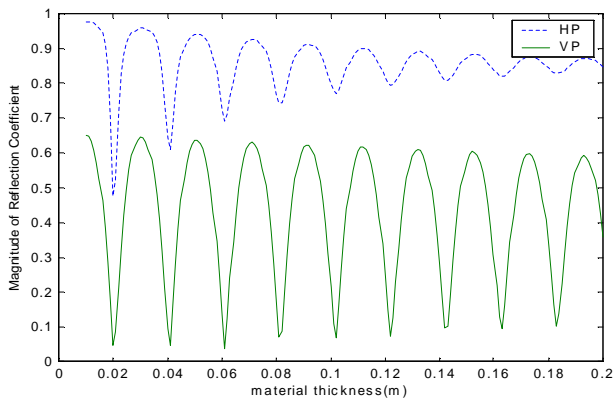

(a)

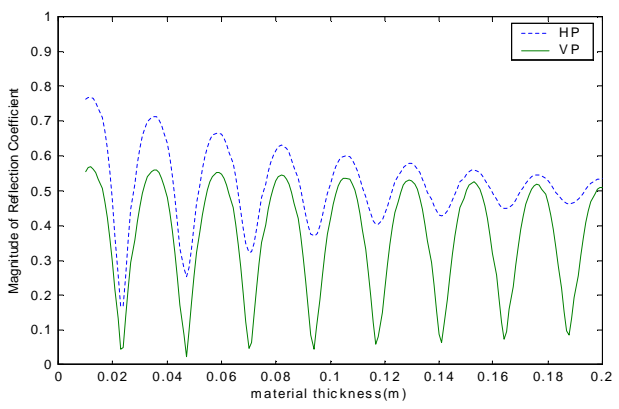

(b)

Fig. (3). Reflection coefficients as a function of the glass material thickness

$$
\text { (a) } \theta=10^{\circ} \text {, (b) } \theta=50^{\circ}
$$




\section{Mohammed: Investigation of the Effect of Building Dielectric Parameters on Indoor}

\section{Ray Tracing Simulation:}

Recently the ray-tracing technique has been used widely to predict radio channel in indoor environments. In this paper we consider the transmitter and receiver both have vertically polarized omidirectional antennas and the antenna pattern prevents strong components from being reflected from the ceiling or the floor. As a result, only the paths from the walls (concrete walls) will contribute significantly to the received signal. To find all the paths under these circumstances, we need only trace the paths in two dimensions.

For the purpose of modeling an $a \times b$ rectangular room is centered at the origin in the $\mathrm{x}-$ $\mathrm{y}$ plane and the transmitter and receiver are placed at $\left(x_{t}, y_{t}\right)$ and $\left(x_{r}, y_{r}\right)$ respectively. The receiver and its images lie at the points $\left((-1)^{n} x_{r}+n a,(-1)^{m} y_{r}+m b\right)$ for integers $n$ and $m$, where $a$ and $b$ are the dimensions of the room. The distance from the transmitter to an image $d_{n m}$ is given by:

$d_{n m}^{2}=\left((-1)^{n} x_{r}+n a-x_{t}\right)^{2}+\left((-1)^{m} y_{r}+m b-y_{t}\right)^{2}$

A ray reaching the $n m t h$ image undergoes $|n|+|m|$ reflections [5]. This simple model was used to predict the indoor channel parameters. Signal level, time dispersion, and coherence bandwidth are predicted and compared by different dielectric parameters under the line of sight situation.

\section{Indoor Channel Parameters:}

Power Level: In indoor environment, the composite received signal is the sums of the signals arriving along different paths. Except for the LOS paths, all paths are going through at least one order of reflection before arriving at the receiver. According to our model mentioned the power in the ray at the $n m$-th image is given by [5].

$P_{n m}=P \frac{\Gamma^{2(n+m)}}{d_{n m}^{2}}$

Where $P$ is constant value, $\Gamma$ represent the reflection coefficient mentioned in equations (1), and (2).

RMS Delay Spread: In wideband system, most of multipath components are resolved and these time-delayed signals contribute to intersymbol interference. RMS delay spread is used to quantify the time dispersion of wideband multipath channel. It gives an indication of maximum data rate that can be achieved without giving rise to intersymbol interference. The RMS delay spread is given by the second moment of impulse response [5, $6]$.

$\tau_{r m s}=\sqrt{\overline{\tau^{2}}-(\bar{\tau})^{2}}$

$\tau_{r m s}$ is the root mean square delay spread

$\bar{\tau}$ is mean excess delay

where, given $L$ propagation paths,

$$
\overline{\tau^{n}} \equiv \frac{\sum_{i=1}^{L} \tau_{i}^{n}\left|P_{i}\right|^{2}}{\sum_{i=1}^{L}\left|P_{i}\right|^{2}} n=1,2
$$


Coherence Bandwidth: A dual representation of delay in terms of a frequency domain parameter is given by the coherence bandwidth $B_{c}$. The coherence bandwidth is inversely proportional to RMS delay spread. Assuming frequency correlation between amplitudes of frequency components being above 0.9 , coherence bandwidth can be approximated by [6].

$$
B_{c} \approx \frac{1}{50 \tau_{r m s}}
$$

\section{Ray Tracing Simulation Results:}

1. Dielectric Constant Effect: Tables (2) and (3) show the effect of increasing the dielectric constants parameters (permittivity and conductivity) on the mean values of power level, rms delay spread, and coherence bandwidth for a given locations of transmitter and receiver. The original values of permittivity and conductivity were taken from [2].

From Table (2) it is found that changes in permittivity value have little effect on the power level, in the other hand variations in RMS delay spread characteristics are observed, indicating the changes in permittivity value have significant impacts on channel RMS delay spread. From Table (3) it is noted that the effect of changes in conductivity values are less significant than from the changes in permittivity values. Most of building structures are low loss materials so that small variations of their conductivity values do not affect the channel characteristics significantly.

Table (2): Channel Parameters for Different Permittivity Values

\begin{tabular}{|c|c|c|c|}
\hline & Original $\varepsilon_{r}$ & $50 \%$ inc & $100 \%$ inc \\
\hline Power Level $(\mathrm{dB})$ & -59.02 & -58.17 & -57.51 \\
\hline$\tau_{r m s}(n s)$ & 13.28 & 14.29 & 15.15 \\
\hline$B_{c}(\mathrm{MHz})$ & 1.50 & 1.39 & 1.32 \\
\hline
\end{tabular}

Table (3): Channel Parameters for Different Conductivity Values

\begin{tabular}{|c|c|c|c|}
\hline & Original $\sigma$ & $50 \%$ inc & $100 \%$ inc \\
\hline Power Level $(\mathrm{dB})$ & -59.02 & -59.01 & -59.00 \\
\hline$\tau_{r m s}(n s)$ & 13.28 & 13.28 & 13.29 \\
\hline$B_{c}(\mathrm{MHz})$ & 1.50 & 1.50 & 1.50 \\
\hline
\end{tabular}

2. Order of Reflection Effect: Simulations was made varying the limit in the order of reflection (the maximum number of reflections that are considered for each ray) and varying the electromagnetic parameters. The influence of the permittivity values in the wide band parameters is more important than the influence of the order of reflection considered for the ray tracing model, the direct and single reflected rays typically represent the more important contribution in amplitude. However, delay parameters are closely related to the order of reflections, the larger this order, the larger time delay.

Table (4) shows the effect of the reflection order on the RMS delay spread for a given two locations of transmitter and receiver, and for original values of dielectric parameters. 
Table (4) RMS delay spread values for different reflection order

\begin{tabular}{|l|c|c|c|}
\hline \multirow{2}{*}{$\tau_{r m s}(n s)$} & \multicolumn{3}{|c|}{ Reflection order } \\
\cline { 2 - 4 } & 2 & 3 & 4 \\
\hline $\begin{array}{l}\text { Location No. } \\
1\end{array}$ & 13.28 & 13.96 & 14.21 \\
\hline $\begin{array}{l}\text { Location No. } \\
2\end{array}$ & 13.23 & 13.77 & 14.01 \\
\hline
\end{tabular}

3. RMS Delay Spread Versus Distance: Fig. 4 shows the fitted plot for RMS delay spread against radial distance between transmitter and receiver for different values of permittivity. It is seen that the RMS delay spread increase with distance from the transmitter up to maximum value that is thereafter constant with distance of the remainder of the room. The maximum value of the delay spread depends mainly on the reflectivity of the enclosing walls and the dimensions of the room.

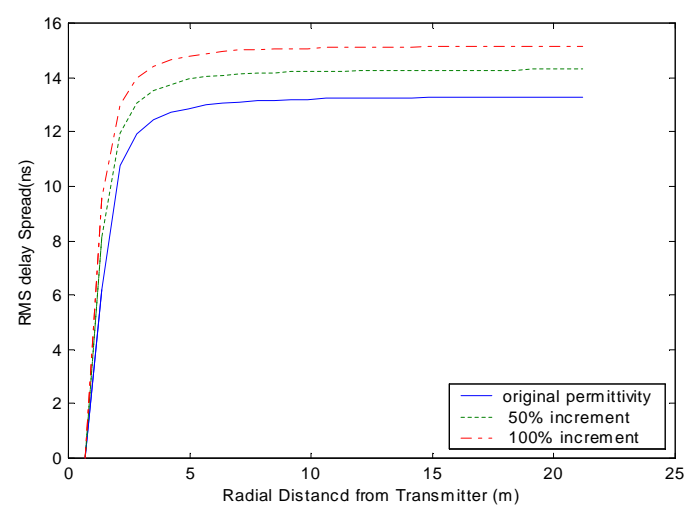

Fig. (4). RMS delay spread against radial distance between transmitter and receiver.

\section{Conclusion:}

The $2.4 \mathrm{GHz}$ reflection coefficients for various smooth building materials as functions of the incidence angle have been calculated. The results show the reflection coefficients in the case of vertical polarization were smaller than those for horizontal polarization, also in the case of vertical polarization the reflection coefficients have values less than 0.1 at a certain incidence angle.

From Fig. 3, it is generally observed that the reflection coefficient of a dielectric material such glass fluctuates when the material is thin, and then converges to a steady value as the material thickness becomes thicker. The reason for this is that the signal decays as it propagates in the dielectric material, and consequently the contribution to the overall reflected signal by the signals multiply reflected inside the material is reduced.

Simulation results have shown that RMS delay spread is more sensitive than power level to building dielectric parameters. The effects of changes in conductivity values are less significant than from the changes in permittivity values.

The influence of electromagnetic parameters is more important than the order of reflection for the ray- tracing model. However, delay parameters are closely related to the order of reflections, the larger this order, the larger time delay.

The RMS delay spread for a given area in (LOS) condition increase with the radial distance from transmitter up to a maximum value, which then remains constant across the remainder of the room. This maximum value depends mainly on the reflectivity of the enclosing walls which depends on the dielectric parameters. 


\section{References:}

[1] T. S. Rappaport, Wireless Communications Principles and Practice, Prentice Hall, 1996.

[2] Y. E. Mohammed, LIU Yuan-an, A. S. Abdallah, "Computation of Dielectric Constants and Loss Tangents at ISM Band by Measurements of Transmission Coefficients", The Journal of China Universities of Posts and Telecommunications, Vol. 10, No. 2, p.p. 6973, Jun. 2003.

[3] L. Boithias, Radio Wave Propagation, North Oxford Academic, 1987.

[4] K. Pahlavan, A. H. Levesque, Wireless Information Networks. New York. John Wiely \& Sons, 1995.

[5] J.T.E. McDonnell, T.P. Spiller, T.A. Wilkinson,"RMS Delay Spread in Indoor LOS Environments at 5.2 GHZ”, Electronics Letters, Vol. 34, No. 11, p.p. 1149-1150, May 1998.

[6] H. J. Zepernic, T. A. Wysocki, "Multipath Channel Parameters for the Indoor Radio at 2.4 GHz ISM Band”, IEEE of Vehicular Technology Conference, Vol. 1, p.p. 190-193, May 1999. 